\title{
Data Security in Dropbox Cloud
}

\author{
Anjali Dange ${ }^{1}$, Satish Sahare ${ }^{2}$, Shubham Amnerkar ${ }^{3}$, Utkarsh Fopare ${ }^{4}$, Vijay V. Chakole ${ }^{5}$ \\ Electronics Department, K.D.K.C.E, Nagpur ${ }^{1,2,3,4,5}$
}

\begin{abstract}
Cloud computing offers a new way of service provision by rearranging various resources over the Internet. The most important and popular cloud service is data storage. In order to preserve the privacy of data holders, data are often stored in cloud in an encrypted form. However, encrypted data introduce new challenges for cloud data deduplication, which becomes crucial for big data storage and processing in cloud. Existing solutions of encrypted data deduplication suffer from security weakness. Therefore, few of them can be readily deployed in practice. In this paper, we propose a scheme to deduplicate encrypted data stored in cloud based on ownership challenge and proxy reencryption. It integrates cloud data deduplication with access control. We evaluate its performance based on extensive analysis and computer simulations. The results show the superior efficiency and effectiveness of the scheme for potential practical deployment, especially for big data deduplication in cloud storage.
\end{abstract}

Keywords: Cloud computing, encryption, data deduplication, cloud computing

\section{INTRODUCTION}

Everyone is talking about the benefits of storing data to the cloud for sharing information among friends, to simplify moving data between different mobile devices, and for small businesses to back up and provide Disaster Recovery (DR) capabilities. But what about the massive amounts of data in enterprise data centers? How do cloud providers protect your data? How is the entire Internet protected? Let's face it; backing up the data from your cellphone to the cloud is fairly routine. The hard job is on the back end, where service providers and large companies need to move, protect and store the massive amounts of data they have within and between their datacenters. If you intend to move large amounts of data over a network and provide access to that data as a service, you need to be cognizant of network bandwidth requirements, data security and the total IT costs of providing those services to end users, especially when providing services for data storage and DR protection. We will examine benefits of deduplication, including the technology computing methods and the implementation types. Data deduplication is one of the hottest technologies in storage right now because it enables companies to save a lot of money on storage costs to store the data and on the bandwidth costs to move the data when replicating it offsite for DR. If you can deduplicate what you store, you can better utilize your existing storage space, which can save money by using what you have more efficiently. If you store less, you also back up less, which again means less hardware and backup media. If you store less, you also send less data over the network in case of a disaster, which means you save money in hardware and network costs over time. The business benefits of data deduplication include:

- Reduced hardware costs;

- Reduced backup costs;

- Reduced costs for business continuity;

- Increased storage efficiency; and

- Increased network efficiency.

\section{AIM AND OBJECTIVE}

In this paper, main aim is to propose a scheme based on data ownership challenge and Proxy Deduplication to manage encrypted data storage with deduplication. We aim to solve the issue of deduplication in the situation where the data holder is not available or difficult to get involved. Specifically, the contributions of this paper can be summarized as below:

We motivate to save cloud storage and preserve the privacy of data holders by proposing a scheme to manage encrypted data storage with deduplication. Our scheme can flexibly support data sharing with deduplication even when the data holder is offline, and it does not intrude the privacy of data holders. We propose an effective approach to verify data ownership and check duplicate storage with secure challenge and big data support. We integrate cloud data deduplication with data access control in a simple way, thus reconciling data deduplication and encryption. We prove the security and assess the performance of the proposed scheme through analysis and simulation. The results show its efficiency, effectiveness and applicability. 


\title{
International Advanced Research Journal in Science, Engineering and Technology
}

\author{
Vol. 6, Issue 2, February 2019
}

Objective is an effective and flexible distributed scheme with explicit dynamic data support to ensure the correctness of users' data in the cloud. whenever data corruption has been detected during the storage correctness verification, our scheme can almost guarantee the simultaneous localization of data errors, i.e., the identification of the misbehaving server(s).

1. Compared to many of its predecessors, which only provide binary results about the storage state across the distributed servers, the challenge-response protocol in our work further provides the localization of data error.

2. Unlike most prior works for ensuring remote data integrity, the new scheme supports secure and efficient dynamic operations on data blocks, including: update, delete and append.

3. Extensive security and performance analysis shows that the proposed scheme is highly efficient and resilient against Byzantine failure, malicious data modification attack, and even server colluding attacks.

\section{PROPOSED WORK}

Data deduplication works by comparing objects (usually files or blocks) and removes objects (copies) that already exist in the data set. All the processes which are not unique are removed in this method.

In Data deduplication method we divide the input data into blocks and a hash value is calculated for each of these blocks. Then using these hash values, we can determine whether another block of same data has already been stored. If a similar data file is found then replace the duplicate data with a reference to the object already present in the database.

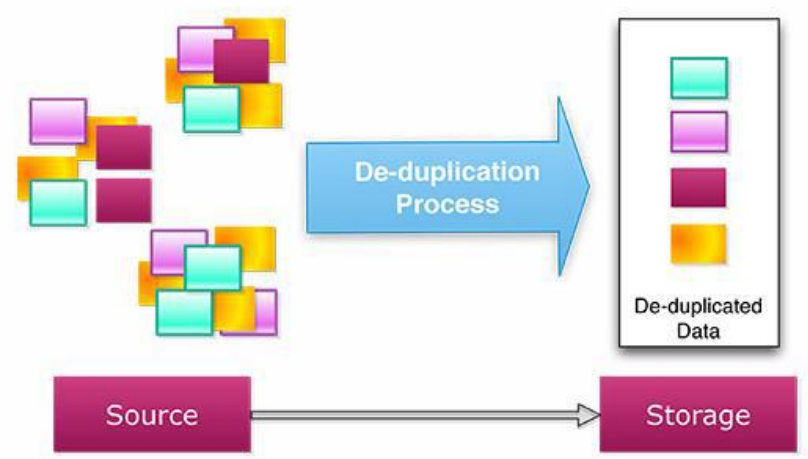

Fig1. Flow Chart (24)

\section{FLOW CHART}

In simplified terms, data deduplication compares objects (usually files or blocks) and removes objects (copies) that already exist in the data set. The deduplication process removes blocks that are not unique. Simply put, the process consists of four steps:

1.Divide the input data into blocks or "chunks."

2.Calculate a hash value for each block of data.

3.Use these values to determine if another block of the same data has already been stored.

4.Replace the duplicate data with a reference to the object already in the database.

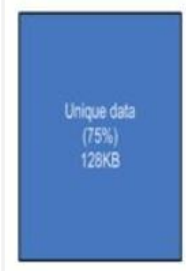

Original block of data is $128 K B$ in size

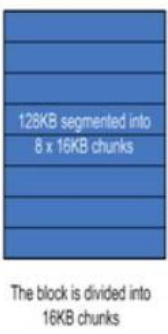

Fig 2. Data Flow Diagram

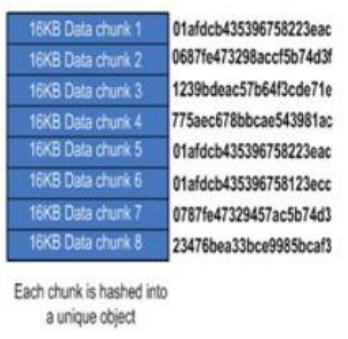

chlchunkis hashed int

Once the data is chunked, an index can be created from the results, and the duplicates can be found and eliminated. Only a single instance of every chunk is stored. The actual process of data deduplication can be implemented in a number of different ways. You can eliminate duplicate data by simply comparing two files and making the decision to delete one that is older or no longer needed. But real deduplication solutions use more sophisticated methods, where the actual math involved can make your head spin. More intelligent file-level deduplication methods actually look inside individual files and compare differences within the files themselves, or compare updates to a file and then just 


\section{International Advanced Research Journal in Science, Engineering and Technology}

Vol. 6, Issue 2, February 2019

store the differences as a "delta" to the original file. File versioning associate's updates to a file and just stores the deltas as other versions. File-based hashing actually creates a unique mathematical "hash" representation of files, and then compares hashes for new files to the original. If there is a hash match, you can guarantee the files are the same, and one can be removed. A lot of backup applications have the versioning capability, and you may have heard it called incremental or differential backup. Some backup software options (Tivoli Storage Manager is a good example) always use the versioning method to speed backup. You do a full back up the first time, and from then on, only the changes in the data need to be stored. IBM calls this "progressive" backup. Other software solutions use similar techniques to reduce Wide Area Network (WAN) requirements for centralized backup. Intelligent software agents running on the client (desktop, laptop or workstation) use file-level versioning or hashing at the client to send only delta differences to a central site. Some solutions actually send all data updates to the central site and then hash the data once it arrives, storing only the unique data elements.

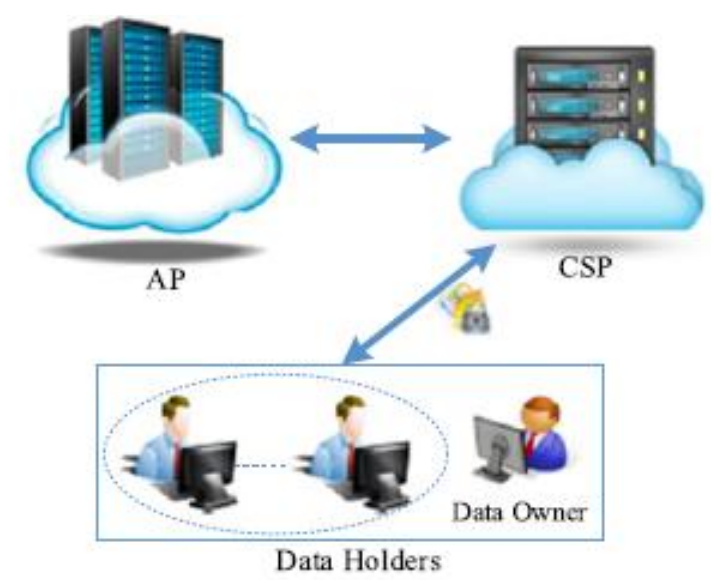

Fig 3 System Architecture

\section{DATA DOWNLOADING ALGORITHM}

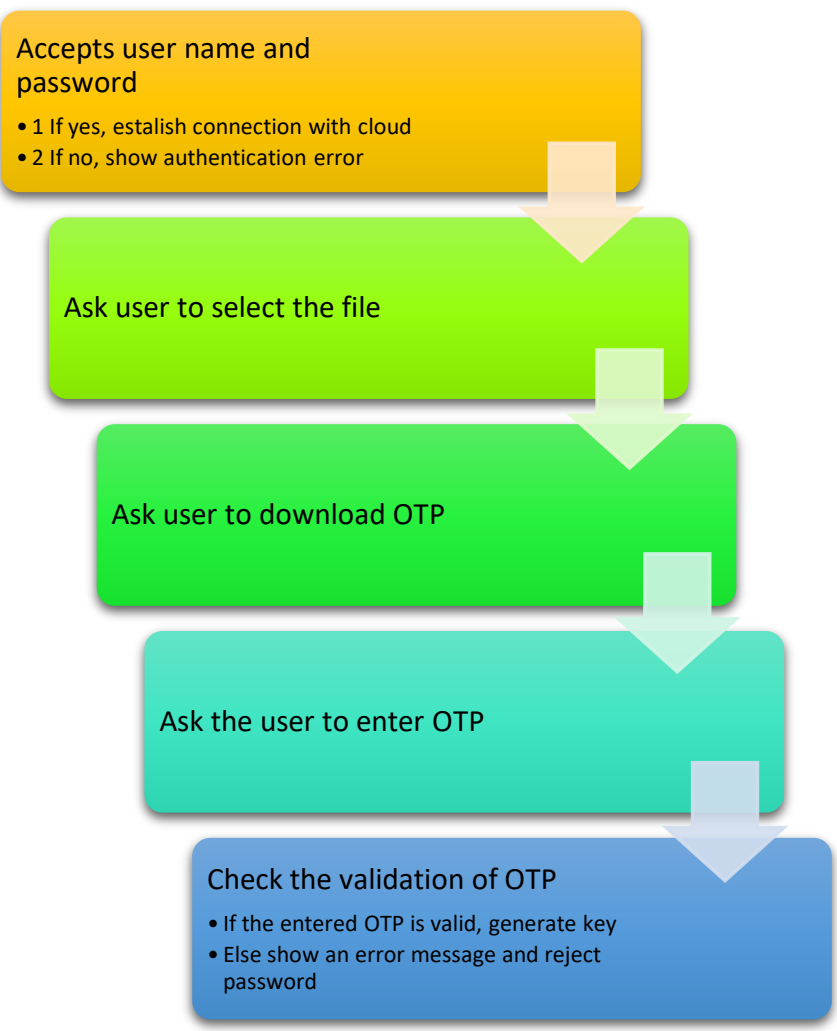




\title{
International Advanced Research Journal in Science, Engineering and Technology
}

\author{
Vol. 6, Issue 2, February 2019
}

\section{CONCLUSION}

In this current work the problem of data security in cloud data storage has been investigated. To ensure the correctness of client data in cloud data storage, the proposed method encrypts data and stores it in cloud and user is allowed for modification of data. Through data security and performance analysis, it shows that work is highly efficient and avoid unauthorized users from accessing the data. With the results and analysis following conclusions are made

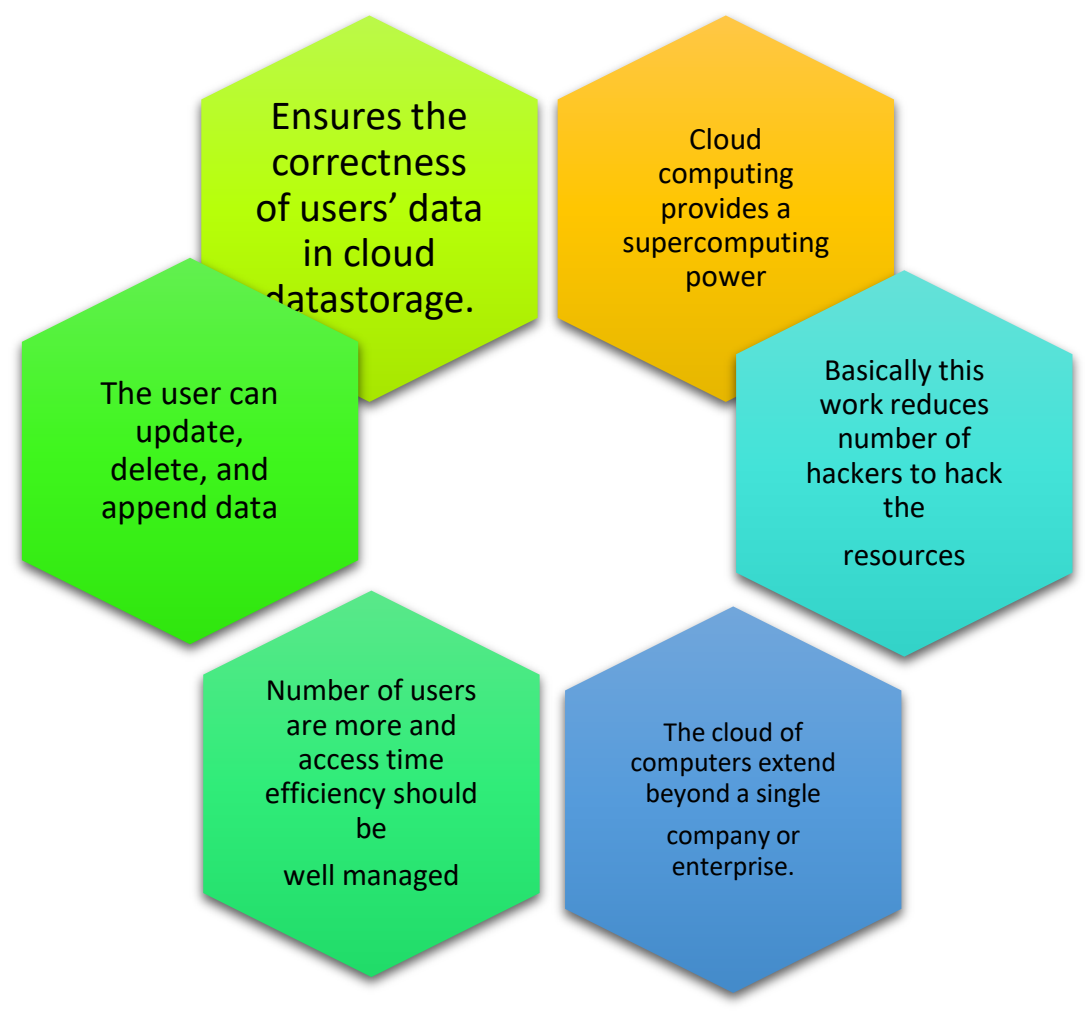

\section{REFERENCES}

[1]. Youssef Gahi, Mouhcine Guennoun, Hussein T. Mouftah,"Big Data Analytics: Security and Privacy Challenges", IEEE Symposium on Computers and Communication (ISCC), Messina, Italy, June 2016, pp 15-17.

[2]. Laila Fetjah, Karim Benzidane, Hassan El Alloussi Othman El Warrak, Said Jai- Andaloussi,” Toward a Big Data Architecture for Security Events Analytic", IEEE $3^{\text {rd }}$ International Conference on Cyber Security and Cloud Computing , Beijing, China,2016,pp 1-7.

[3]. Natalia Miloslavskaya and Aida Makhmudova, "Survey of Big Data Information Security", 4th International Conference on Future Internet of Things and Cloud Workshops, Vienna, Austria, Aug 2016,pp 4-9.

[4]. Suliman A. Alsuhibany," A Space-and-Time Efficient Technique for Big Data Security Analytics", vol. 46, no. 2, Riyadh, Saudi Arabia ,pp.241-284, 2016.

[5]. Hai-ting Cui, "Research on the Model of Big Data Serve Security in Cloud Environment", First IEEE International Conference on Computer Communication and the Internet, Wuhan, China,Oct 2016, pp 1-16.

[6]. Cong Wang, Qian Wang, and Kui Ren," Ensuring Data Storage Security in Cloud Computing”, US National Science Foundation,2015, pp 1-4

[7]. Raj Kumar, "Research on Cloud Computing Security Threats using Data Transmission" International Journal of Advanced Research in Computer Science and Software Engineering, India Volume 5, Issue 1, January 2015, pp. 399-402.

[8]. Saakshi Narula," Cloud computing security: Amazon web service", Fifth International Conference on Advanced Computing \& Communication Technologies, Haryana, India,Feb 2015,pp 699-703

[9]. Changyou Guo and Xuefeng Zheng "The Research of Data Security Mechanism Based on Cloud Data Storage Architecture", International Journal of Security and Its Applications, China, Vol. 9, No. 3 (2015), pp. 363-370

[10]. Ahmed Dheyaa Basha, Irfan Naufal Umar, and Merza Abbas," Storage in cloud as a module", International Journal .30,Vol. 4, No. 1, January 2014, pp 160-163

[11]. Yunchuan Sun, and Junsheng Zhang," Data Security and Privacy in Cloud Computing", International Journal of Distributed Sensor Networks Japan, Volume 5,2014,pp 9-20.

[12]. Buyya," Introduction to module interactions in a system on Cloud Computing”,IEEE transactions on cloud computing, Italy, vol. 1, no. 1, january-june 2013,pp 2424-2444

[13]. Zohreh Sanaei, "Heterogeneity in Cloud Computing: Taxonomy and Open system modules",Bellingham, IEEE Communications Surveys \& Tutorials, Vol. 16, No. 1, 2013, pp 40-45.

[14]. Young-Sae Song,"Storing Big Data- The Rise of the Storage Cloud",Maldives,pp. 504- 511, 2012.

[15]. D. L. G. Filho and P. S. L. M. Barreto, "Demonstrating Data Possession and Uncheatable Data Transfer" Italy, Cryptology ePrint Archive, Volume 78, Issue 5, September 2012, pp 1345-1358

[16]. Intel IT center, "Peer Research Big Data Analytics "Intel's IT Manager Survey on How Organizations Are Using Big Data”, USA, Aug 2012, pp 46-55. 


\section{International Advanced Research Journal in Science, Engineering and Technology}

Vol. 6, Issue 2, February 2019

[17]. Vahid Ashktorab and Dr. Kamran Zamanifar ," A Survey on Cloud Computing and Functional Solution Providers", International Journal of Application or Innovation in Engineering \& Management (IJAIEM), Iran ,Vol 1, issue 2,Oct 2012, pp 1191-1201.

[18]. T. S. J. Schwarz and E. L. Miller, "Store, Forget, and Check: Using Algebraic Signatures to Check Remotely Administered Storage," Egypt, Proc. of ICDCS '06, pp. 12-12, 2011

[19]. I.Somerville, Software engineering, 9th edition, vol.ISBN 97801370 35151.Adison Wisley publishing company, 2010

[20]. Q. Wang, K. Ren, W. Lou, and Y. Zhang, "Dependable and Secure Data Storage Architecture," Rio de Janeiro, Brazil, Apr 2009 , pp 688-692.

[21]. G. Ateniese, R. D. Pietro, L. V. Mancini, and G. Tsudik,"Scalable and Efficient Provable Data Possession," Istanbul, Turkey Proc. of SecureComm '08, pp. 1-10, 2008.

[22]. K. D. Bowers, A. Juels, and A.Oprea, "HAIL: A High-Availability and Integrity Layer for Cloud Storage," Cryptology ePrint Archive, USA, 2008, pp 102-113.

[23]. R. Curtmola, O. Khan, R. Burns, and G. Ateniese, “MRPDP: Multiple- Replica Provable Data Possession”, USA, pp. 411-420, 2008.

[24]. https://goo.gl/images/c4eDez 